\title{
SOME EXACT RESULTS FOR QUANTUM LATTICE PROBLEMS
}

\author{
J. CHRIS EILBECK \\ Department of Mathematics, \\ Heriot-Watt University, \\ Edinburgh EH14 4AS, UK \\ E-mail: J.C.Eilbeck@hw.ac.uk
}

\begin{abstract}
We study the exact eigenvalue spectrum for a variety of quantum lattice models, concentrating on the Quantum Discrete Nonlinear Schrödinger (QDNLS) model. In particular we discuss eigenstates which are the quantum equivalent of the classical breather. These results extend previous work, by considering larger number of quanta and bigger lattices.
\end{abstract}

\section{Introduction}

Physics is increasingly moving towards nano-scale technology, and with this comes the need to understand small lattices or quasi-lattices supporting a small number of quanta. Often this gives the quantum equivalent of a classical breather, though with some interesting differences. In this short paper we review some work on quantum lattice problems, concentrating mainly on the quantum discrete nonlinear Schrödinger (QDNLS) model. The corresponding classical DNLS lattice is discussed elsewhere in this book. Our eventual aim is to understand such systems as quantum dot arrays, BoseEinstein Condensate lattices, and even models for Quantum Computers.

We adopt here a pedagogical approach with many details omitted$\mathrm{see}^{2,5,6}$ for a fuller description of the background material in our approach, and ${ }^{3,4}$ for some other interesting developments by other authors.

Consider the classical DNLS model ${ }^{1}$ in one spatial dimension with nearest-neighbour interactions

$$
\mathrm{i} \frac{d A_{j}}{d t}+A_{j-1}+A_{j+1}+\gamma\left|A_{j}\right|^{2} A_{j}=0,
$$

where $j=1,2, \ldots, f$ are the lattice points, the $A_{j}$ 's are the complex mode amplitudes at each site $j$, and $\gamma$ is the anharmonic parameter. This 
equation can be derived from the following Hamiltonian

$$
H=-\sum_{j=1}^{f}\left[\frac{1}{2} \gamma\left|A_{j}\right|^{4}+A_{j}^{*}\left(A_{j+1}+A_{j-1}\right)\right],
$$

where the canonical variables are $A_{j}, A_{j}^{*}$, and periodic boundary conditions are assumed $\left(A_{j+f}=A_{j}\right)$. As shown elsewhere in this volume, this model supports both stationary and mobile breathers, strongly localized solutions with an internal mode of oscillation. How do these properties carry over to the quantum case?

A corresponding Quantum DNLS Hamiltonian is

$$
\hat{H}=-\sum_{j=1}^{f}\left[\frac{\gamma}{2} b_{j}^{\dagger} b_{j}^{\dagger} b_{j} b_{j}+b_{j}^{\dagger}\left(b_{j-1}+b_{j+1}\right)\right]
$$

where the boson annihilation $\left(b_{j}\right)$ and creation $\left(b_{j}^{\dagger}\right)$ operators destroy or create a boson at site $j$ according to the following rules.

$$
b_{j}\left|n_{j}\right\rangle=\sqrt{n_{j}}\left|n_{j}-1\right\rangle, \quad b_{j}|0\rangle=0, \quad b_{j}^{\dagger}\left|n_{j}\right\rangle=\sqrt{n_{j}+1}\left|n_{j}+1\right\rangle
$$

where $\left|n_{j}\right\rangle$ is the number of bosons at lattice point $j$. The $b_{j}$ satisfy the boson commutation relations $b_{j} b_{k}^{\dagger}-b_{j}^{\dagger} b_{k}=\delta_{j, k}$

An important feature is that the Hamiltonian (1) conserves the number of bosons in the system

$$
\hat{N}=\sum_{j=1}^{f} b_{j}^{\dagger} b_{j} .
$$

The methods we discuss can be extended to a range of other numberconserving models, for example the following boson models

- The Quantum Ablowitz-Ladik (QAL) model

$$
\hat{H}=-\sum_{j=1}^{f}\left[a_{j}^{\dagger}\left(a_{j+1}+a_{j-1}\right)\right],
$$

where $a_{j}^{\dagger}$ and $a_{j}$ are operators satisfying the commutation relations $\left[a_{j}^{\dagger}, a_{k}^{\dagger}\right]=\left[a_{j}, a_{k}\right]=0,\left[a_{j}, a_{k}^{\dagger}\right]=\left(1+\frac{1}{2} \gamma a_{j}^{\dagger} a_{k}\right) \delta_{j k}$.

- The Salerno system. This is a $q$-deformation of the QDNLS system which interpolates between the QAL and QDNLS systems. Now $\left[a_{j}, a_{k}^{\dagger}\right]=\left(1+\frac{1}{2}(\gamma-\epsilon) a_{j}^{\dagger} a_{k}\right) \delta_{j k}$, and the Hamiltonian is

$$
\hat{H}=-\sum_{j=1}^{f}\left[a_{j}^{\dagger}\left(a_{j+1}+a_{j-1}\right)-\right.
$$




$$
\left.-\frac{2 \epsilon}{(\gamma-\epsilon)}\left(\frac{\ln \left(1+\frac{1}{2}(\gamma-\epsilon) a_{j}^{\dagger} a_{j}\right)}{\ln \left(1+\frac{1}{2}(\gamma-\epsilon)\right)}+a_{j}^{\dagger} a_{j}\right)\right] .
$$

Note that the corresponding number operator will be different in all these cases, details can be found in ${ }^{6,5}$. The methods also extend to a number of fermion models such as a fermionic polaron model and the Hubbard model.

\section{Eigenvalues of the QDNLS Hamiltonian}

We now describe our computational method. Since the number is conserved, we can block-diagonalize the Hamiltonian matrix using states which are simultaneously eigenstates of $\hat{H}$ and $\hat{N}$

$$
H=\langle\Psi|\hat{H}| \Psi\rangle=\left(\begin{array}{ccccc}
H_{0} & 0 & & & \\
0 & H_{1} & 0 & & \\
& 0 & H_{2} & 0 & \\
& & \ddots & \ddots & \ddots
\end{array}\right)
$$

where $H_{n}$ is the block describing states with a total of $n$ bosons. Each eigenstate for a fixed value of $n$ is formed as a linear combination of number states with a fixed $n$.

$$
\left|\Psi_{n}\right\rangle=\sum_{i} c_{i}\left|\psi_{n}^{(i)}\right\rangle
$$

The number states $\left|\psi_{n}^{(i)}\right\rangle$ are formed from the different ways we can distribute $n$ bosons over the $f$ sites on the lattice (we are assuming a finite lattice with periodic boundary conditions).

$$
\left|\psi_{n}^{(i)}\right\rangle=\left|n_{1}^{(i)}\right\rangle\left|n_{2}^{(i)}\right\rangle \ldots\left|n_{f}^{(i)}\right\rangle=\left[n_{1}^{(i)}, n_{2}^{(i)}, \ldots, n_{f}^{(i)}\right] \text {, where } n=\sum_{j} n_{j}^{(i)} .
$$

For example, $[2,2,0,0,0,1]$ means a state with 2 bosons on site 1,2 bosons on site 2 , and 1 boson on site 6 . For a fixed value of $n$ and $f$ there are $(n+f-1) ! / n !(f-1) !$ different number states, a quantity which expands rapidly with $n$ and $f$.

We can further block-diagonalize the Hamiltonian by using the fact that it is translationally invariant, and hence we can simultaneously diagonalize with respect to the momentum operator ${ }^{5,6}$. As a simple example, consider a 1 D periodic lattice of length $f=3$ with $n=2$ bosons. There are 6 possible number states $[2,0,0],[0,2,0],[0,0,2],[1,1,0],[0,1,1],[1,0,1]$, so $\mathrm{H}_{2}$ in this case is $6 \times 6$. But we can block-diagonalize this into three $2 \times 2$ 
blocks $H_{2, k}$ using the translationally invariant states

$$
\begin{aligned}
& \left|\Psi_{2}^{(a)}\right\rangle=[2,0,0]+t[0,2,0]+t^{2}[0,0,2] \\
& \left|\Psi_{2}^{(b)}\right\rangle=[1,1,0]+t[0,1,1]+t^{2}[1,0,1]
\end{aligned}
$$

with $t=1, \exp 2 \pi i / 3, \exp -2 \pi i / 3$, so that $t^{3}=1$, with corresponding $k$ (momentum) values $0, \pm 2 \pi / 3$ respectively. When $f$ and $n$ are large this can give a substantial saving in calculation time.

The problem of a non-translationally invariant $\hat{H}$, such as a lattice with a defect, is a more difficult problem and is currently under investigation.

\subsection{Quantum Mechanics in Maple}

We can further speed up our studies by using an algebraic manipulation package to manipulate the states and to calculate the Hamiltonian in algebraic form as a function of the parameters. In Maple, for example, we represent $[2,2,0,0,0,1]$ as psi $(2,2,0,0,0,1)$, where psi() is an "undefined" function. Then the operators $b_{j}^{\dagger}$ are defined something like

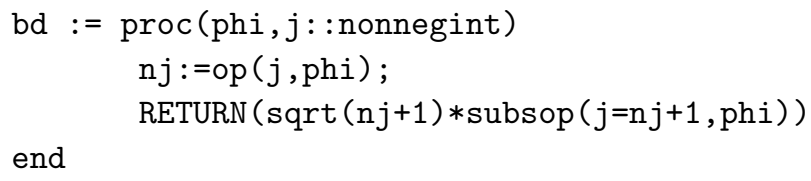

with a corresponding definition for $b$, the QDNLS $\hat{H}$ is defined along the following lines

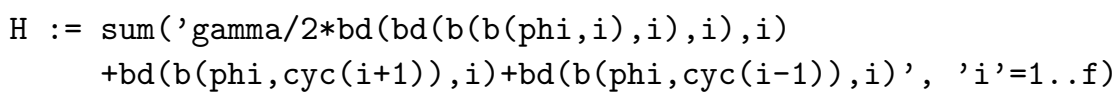

where cyc deals with the periodic boundary conditions, i.e. $c y c(f+1)=1$, etc. This is only a brief sketch of the codes lying behind the calculations. It has recently been found that by careful optimization of the algorithms, a speedup of almost two orders of magnitude is possible. This, combined with the continuing improvement in micro-chip speeds, means that much bigger problems can be tackled than 10 years ago. 


\subsection{The $n=2$ case}

In this case each $H_{2, k}$ is tridiagonal. In the case of the QDNLS equation for large odd $f$, the value of $H_{2, k}$ is given by

$$
H_{2, k}=\left(\begin{array}{ccccccc}
\gamma & \sqrt{2} q^{*} & & & & \\
\sqrt{2} q & 0 & q^{*} & & & \\
& q & 0 & q^{*} & & \\
& & q & 0 & q^{*} & \\
& & & \ddots & \ddots & \ddots
\end{array}\right),
$$

where $q=-(1+\exp (i k))$. Eigenvalues and eigenvectors can be calculated numerically, or analytically, and some simple formula are known in the limit $f \rightarrow \infty$. An investigation of the $1 \mathrm{D}$ case for various models was made in 1992 by Eilbeck and Pego, but unfortunately this work has not yet been published. The results in the limit $f \rightarrow \infty$ for the QDNLS model are shown in Fig.1. In this figure, eigenvalues are plotted vertically for each $k$ value

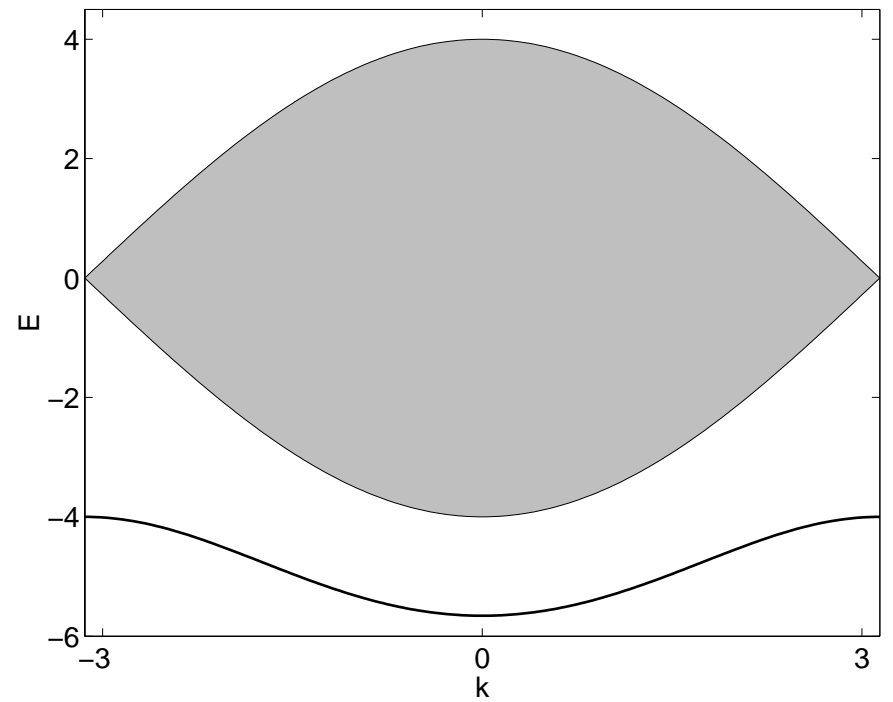

Figure 1. Eigenvalues $E(k)$ for QDNLS, $n=2, \gamma=4$. The lower band is the "breather" band.

on the horizontal axis. There is a continuum set and an isolated eigenvalue for each $k$. The equation for the lower band of isolated eigenvalues is, in the limit $f \rightarrow \infty$,

$$
E=-\sqrt{\gamma^{2}+16 \cos ^{2}(k / 2)}
$$


The isolated band corresponds to an eigenvector of $H_{2, k}$ with the following form

$$
v=\left(\frac{1}{\sqrt{2}}, \mu, \mu^{2}, \mu^{3}, \ldots\right)^{\prime}
$$

where

$$
\mu=\frac{-(\gamma+E) e^{i k / 2}}{4 \cos (k / 2)} .
$$

This eigenvalue and the corresponding eigenvector can be checked by direct calculation. It is also straightforward to check that $|\mu|^{2}<1$ for $\gamma>0$. When $\gamma \gg \cos (k / 2)$ we have

$$
\mu \approx \frac{2}{\gamma} \cos (k / 2) \exp (i k / 2)
$$

so that $\mu \rightarrow 0$ as $\gamma \rightarrow \infty$. Note also that $\mu=0$ when $k= \pm \pi$, a result originally pointed out by S. Flach (private communication).

The ordering is such that the $i$ th element of $v$ multiplies the following translationary invariant states $\Psi_{i}$

$$
\begin{aligned}
\Psi_{1} & =[2,0,0, \ldots]+e^{i k}[0,2,0, \ldots]+e^{2 i k}[0,0,2, \ldots]+\ldots \\
\Psi_{2} & =[1,1,0, \ldots]+e^{i k}[0,1,1,0, \ldots]+e^{2 i k}[0,0,1,1,0, \ldots]+\ldots \\
\Psi_{3} & =[1,0,1,0, \ldots]+e^{i k}[0,1,0,1,0, \ldots]+e^{2 i k}[0,0,1,0,1,0, \ldots]+\ldots \\
\ldots & =\ldots
\end{aligned}
$$

so that the (unnormalized) eigenfunction is

$$
\Psi=\sum_{i=1}^{\infty} v_{i} \Psi_{i}
$$

This is a localized eigenfunction in the sense that there is a high probability of finding the two bosons on the same site, but with an equal probability of finding these two bosons at any site in the system. We claim this is a quantum analogue of the classical localized breather.

\subsection{Results for general $n, \gamma$}

There are some general results for general $n$, but only in the large $\gamma$ limit $^{6,5}$. In this case there is still a "breather" band with eigenfunction

$\Psi \approx[n, 0,0, \ldots]+[0, n, 0, \ldots]+\ldots+[0,0, \ldots, n]+O\left(\gamma^{-1}\right)([n-1,1,0, \ldots]+\ldots)$, 
but the continuum band in the $n=2$ case now bifurcates into a number of separate bands. These can be understood by considering the $\gamma$-dependent term in the Hamiltonian

$$
-\sum_{j=1}^{f}\left[\frac{\gamma}{2} b_{j}^{\dagger} b_{j}^{\dagger} b_{j} b_{j}\right] .
$$

Any site with $n_{j}>1$ will contribute a value $\frac{1}{2} \gamma n_{j}\left(n_{j}-1\right)$ to this sum. If all the bosons are on one site this gives $\frac{1}{2} \gamma n(n-1)$. If there are $n-1$ bosons at one site and 1 at another site, the contribution will be $\frac{1}{2} \gamma(n-1)(n-2)$. If there are $n-2$ bosons at one site and 2 at another site, the contribution will be $\frac{1}{2} \gamma[(n-2)(n-3)+2]$, and so on.

For example, consider the case $n=4$. With $\gamma=0$ we get the spectrum shown in Fig.2. Note that now we are working with a finite size lattice, the spectrum is for discrete $k$ values, but it is clear that in the continuum limit we will get a single continuum band. If we now increase $\gamma$ to 7 we get Fig.3.

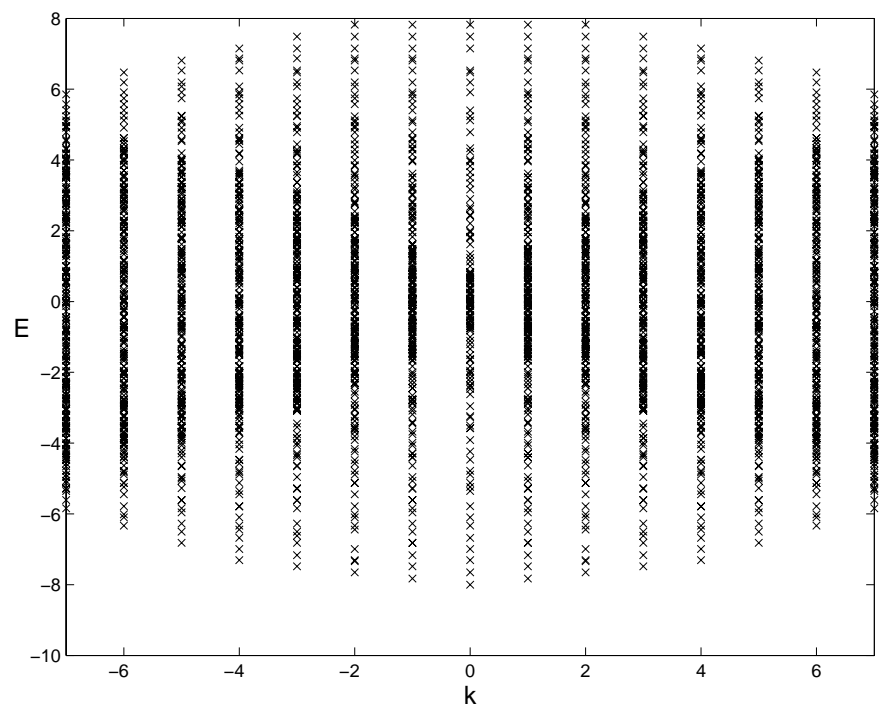

Figure 2. Example: 4 bosons, 15 sites, $\gamma=0$

Now the single breather band has clearly split off below, and the main continuum band has started to split into two or more bands. If we increase $\gamma$ to 14 we get Fig.4. Now there are 5 bands clearly visible. The lowest corresponds to the single 4-breather band, conveniently labelled (in the 


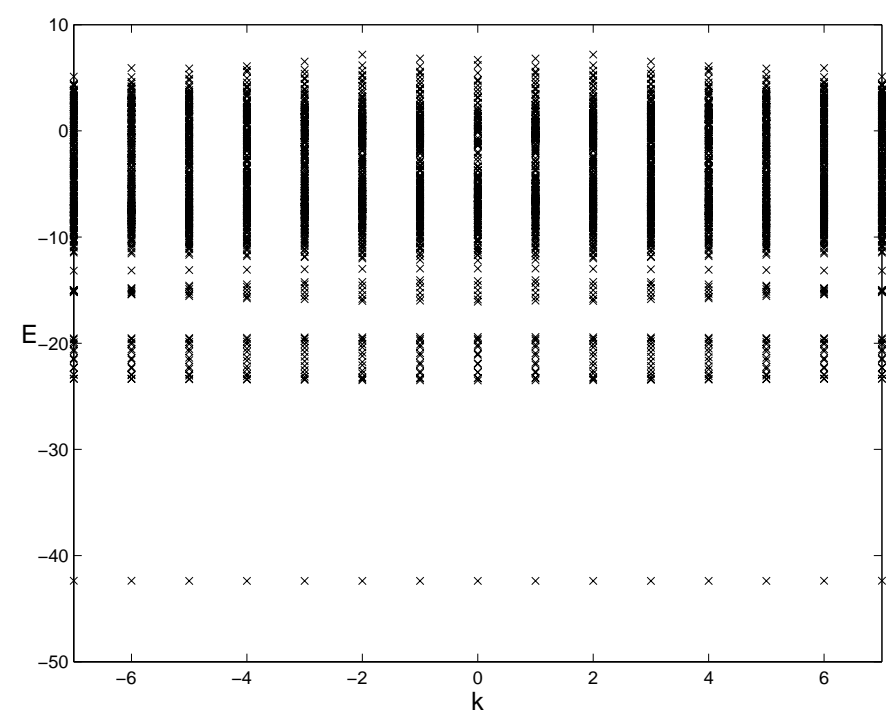

Figure 3. Example: 4 bosons, 15 sites, $\gamma=7$

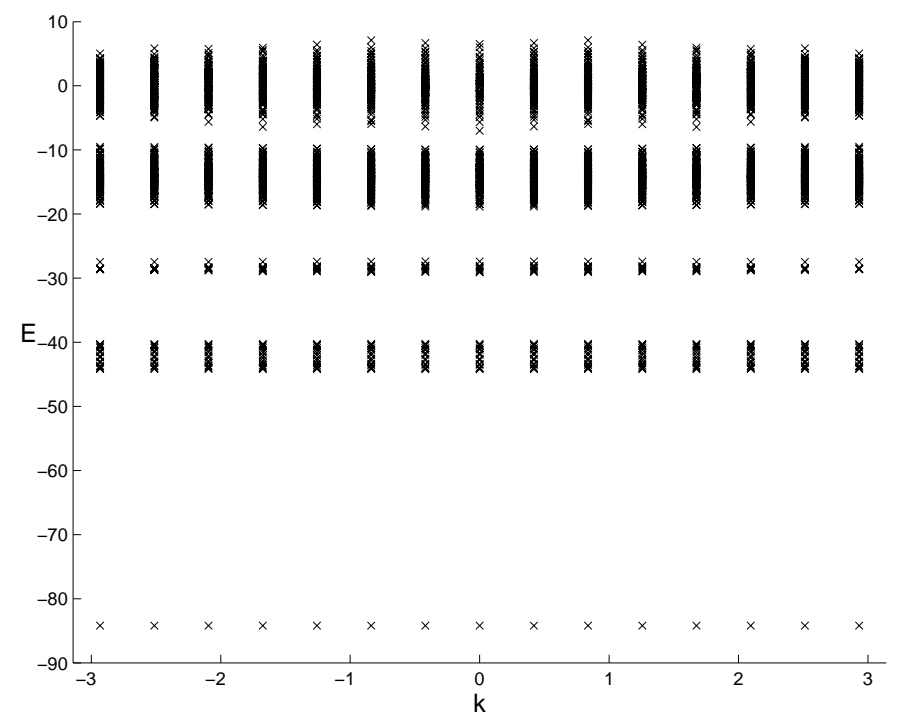

Figure 4. Example: 4 bosons, 15 sites, $\gamma=14$ 
large $\gamma$ limit) as $[4,0, \ldots]$ (plus cyclic permutations). The next lowest is the "3-breather band plus single boson" band $[3,1,0, \ldots]$, plus permutations. The next narrow band is the "double 2-breather band" $[2,2,0, \ldots]$. Interestingly, this band shows some structure at higher magnification which will be reported elsewhere. Moving up, the penultimate band is the "2-breather band plus single bosons", $[2,1,1,0, \ldots]$, and the top band consists of single bosons only, $[1,1,1,1,0, \ldots]$.

As a final example, we show the result of a calculation on a $2 \mathrm{D}$ lattice with $n=2$. Fig.5. The "breather band" is now a 2 -dimensional sheet, and

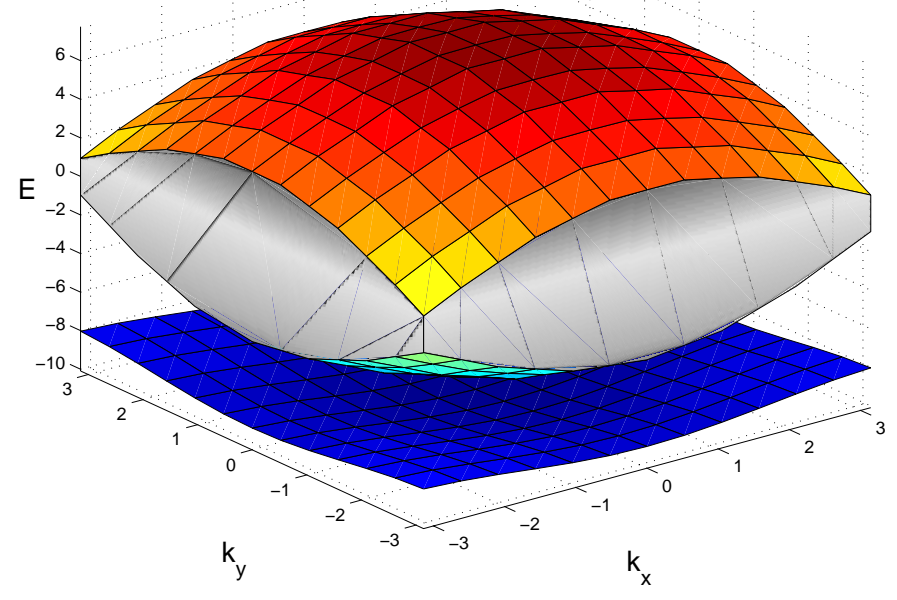

Figure 5. Example: 2 bosons, $13 \times 13$ lattice

the "continuum band" is a lens-shaped volume.

Further work is now concentrating on the fine structure of these bands in one and higher dimensions, both in QDNLS and in QAL and other models.

\section{Acknowledgements}

I am grateful for support from the EU under the LOCNET grant. I would also like to thank Oliver Penrose, Mario Salerno and Alwyn Scott for many helpful discussions. 


\section{References}

1. J. C. Eilbeck, P. S. Lomdahl, and A. C. Scott. The discrete self-trapping equation. Physica D, 16:318-338, 1985.

2. J. C. Eilbeck and A. C. Scott. Quantum lattices. In K. H. Spatschek and F. G. Mertens, editors, Nonlinear Coherent structures in Physics and Biology, NATO ASI Series B: Physics, volume 329, pages 1-14. Plenum Press, 1994.

3. S. Flach and V. Fleurov. Tunnelling in the nonintegrable trimer - a step towards quantum breathers. J. Phys.-Cond. Matt., 9:7039-7061, 1997.

4. R. S. MacKay. Discrete breathers: classical and quantum. Physica A, 288:174198, 2000.

5. A. C. Scott. Nonlinear Science. OUP, Oxford, 1999.

6. A. C. Scott, J. C. Eilbeck, and H. Gilhøj. Quantum lattice solitons. Physica D, 78:194-213, 1994. 